\title{
Lyapunov, Floquet, and singular vectors for baroclinic waves
}

\author{
R. M. Samelson \\ College of Oceanic and Atmospheric Sciences, 104 Ocean Admin Bldg, Oregon State University, Corvallis, OR, USA
}

Received: 18 September 2000 - Accepted: 29 January 2001

\begin{abstract}
The dynamics of the growth of linear disturbances to a chaotic basic state is analyzed in an asymptotic model of weakly nonlinear, baroclinic wave-mean interaction. In this model, an ordinary differential equation for the wave amplitude is coupled to a partial differential equation for the zonal flow correction. The leading Lyapunov vector is nearly parallel to the leading Floquet vector $\phi_{1}$ of the lowest-order unstable periodic orbit over most of the attractor. Departures of the Lyapunov vector from this orientation are primarily rotations of the vector in an approximate tangent plane to the large-scale attractor structure. Exponential growth and decay rates of the Lyapunov vector during individual Poincaré section returns are an order of magnitude larger than the Lyapunov exponent $\lambda \approx 0.016$. Relatively large deviations of the Lyapunov vector from parallel to $\phi_{1}$ are generally associated with relatively large transient decays. The transient growth and decay of the Lyapunov vector is well described by the transient growth and decay of the leading Floquet vectors of the set of unstable periodic orbits associated with the attractor. Each of these vectors is also nearly parallel to $\phi_{1}$. The dynamical splitting of the complete sets of Floquet vectors for the higher-order cycles follows the previous results on the lowest-order cycle, with the vectors divided into wavedynamical and decaying zonal flow modes. Singular vectors and singular values also generally follow this split. The primary difference between the leading Lyapunov and singular vectors is the contribution of decaying, inviscidly-damped wave-dynamical structures to the singular vectors.
\end{abstract}

\section{Introduction}

The predictability of geophysical fluid flows is an important and interesting scientific issue, which combines practical and theoretical elements. Of particular practical interest is the problem of numerical weather prediction. One ap-

Correspondence to: R. M. Samelson

(rsamelson@coas.oregonstate.edu) proach to this problem involves the use of ensemble forecasting techniques, which attempt to improve a single atmospheric forecast by combining multiple model predictions (Epstein, 1969; Leith, 1974).

Recent operational implementations of ensemble forecasting in global numerical weather prediction models rely on two different methods for ensemble generation: bred modes (Toth and Kalnay, 1997) and singular vectors (Buizza et al., 1993; Ehrendorfer and Tribbia, 1997). Bred modes are obtained by iterating a "breeding" cycle, in which the differences between ensemble members and a control forecast are rescaled and added to the analysis at each analysis cycle to initialize a new ensemble. Singular vectors are optimal disturbances (Lorenz, 1965; Farrell, 1989) that maximize specific measures of disturbance growth over specific forecast intervals.

The object of the present contribution is to compute and analyze singular vectors and the simplest analogs of bred modes in a simple, physically consistent model of baroclinic wave-mean interaction, in order to develop insight into the processes of disturbance growth in time-dependent baroclinic flows and their relation to ensemble forecasting methods. The present study shares this general motivation with the closely related study of Samelson (2001; hereafter S2001), which it extends and as a companion to which it should be read, and with many recent studies of systems ranging in complexity from the low-order Lorenz (1963) equations to operational numerical weather prediction models (e.g. Buizza and Palmer, 1995; Buizza, 1995; Trevisan and Legnani, 1995; Legras and Vautard, 1996; Szunyogh et al.,1997; Vannitsem and Nicolis, 1997).

The dynamics considered here and by S2001 are the asymptotic wave-mean interaction equations derived by Pedlosky (1971) and studied further by Pedlosky and Frenzen (1980) and Klein and Pedlosky (1986). These equations describe the evolution of a weakly nonlinear baroclinic wave and a zonal flow correction for a zonal flow near marginal stability, and represent the simplest physical model of nonlinear baroclinic dynamics that is currently available. 
The present study extends S2001, which focused on disturbances to stable and unstable time-periodic solutions of the model equations, to consider dirsturbances to irregular, chaotic solutions. Since the pioneering work of Lorenz (1963), deterministic chaos has served as an enduring metaphor for the observed irregularity and unpredictability of the atmosphere. One goal of this study is, in a limited way and for one particular physical model, to explore this metaphor quantitatively and concretely, in the context of ensemble forecasting. The approach is partially motivated by recent work on cycle expansions for chaotic systems (e.g. Artuso et al., 1990a, 1990b; Christiansen et al., 1997; Cvitanović et al., 2000), and is related to a recent study based on the Lorenz system (Trevisan and Pancotti, 1998).

The model is briefly reviewed in Sect. 2. Section 3 describes the chaotic basic state and its linear instability, and Sects. 4 and 5 discuss the Floquet and singular vector analyses, respectively. Section 6 contains the discussion, and Sect. 7 summarizes the results.

\section{Model}

The model studied here is a two-layer, $f$-plane, quasigeostrophic fluid in a periodic channel with a rigid lid at the upper boundary, and Ekman dissipation at both the upper and lower boundaries. Weakly nonlinear baroclinic wave-mean interaction has been studied in this model by Pedlosky (1971) and Pedlosky and Frenzen (1980), and is summarized in Pedlosky (1987). The model equations and the relevant parameters are summarized here, with notation primarily following the previous references, to which the reader is referred for additional details.

For a weakly supercritical mean flow, a weakly nonlinear disturbance consisting of a single zonal wave component generates a small correction to the mean zonal flow, which, in turn, affects the growth or decay of the wave. The asymptotic analysis conducted by Pedlosky (1971) yields the coupled system of equations describing this interaction. This system consists of a second-order ordinary differential equation for the wave amplitude $A(t)$, coupled to a partial differential equation for the mean flow correction $\Psi(y, t)$. If $\Psi$ is expanded in terms of sinusoidal cross-channel modes, the partial differential equation transforms into an infinite set of coupled ordinary differential equations, which may be written as

$$
\begin{aligned}
\frac{d A}{d t} & =-\gamma A+B, \\
\frac{d B}{d t} & =-\frac{1}{2} \gamma B+A\left[1+\frac{1}{2} \gamma^{2}-\sum_{j=1}^{J} a_{j}\left(A^{2}+V_{j}\right)\right], \\
\frac{d V_{j}}{d t} & =-\gamma\left(b_{j} V_{j}-c_{j} A^{2}\right), \quad j=1,2, \ldots,
\end{aligned}
$$

where $J \rightarrow \infty$ for the complete expansion, and

$a_{j}=\frac{32 m^{2}(2 j-1)^{2}}{\left[(2 j-1)^{2}-4 m^{2}\right]^{2}\left[(2 j-1)^{2} \pi^{2}+K^{2}\right]}$,

$$
\begin{aligned}
b_{j} & =\frac{(2 j-1)^{2} \pi^{2}}{(2 j-1)^{2} \pi^{2}+K^{2}}, \\
c_{j} & =2-b_{j} .
\end{aligned}
$$

Here, $K=\left(k^{2}+m^{2} \pi^{2}\right)^{1 / 2}$ is the total wave number of the baroclinic wave, and $\gamma=r /(2 \sigma)$ is the Ekman damping coefficient $\varepsilon r$ scaled by twice the inverse time scale, the small exponential growth rate $\varepsilon \sigma$ of the linear wave. The relations between $A, B, V_{j}$ and the upper and lower layer stream functions are discussed in S2001. Briefly, $A$ is the scaled amplitude of the wave, $B$ is a measure of the phase shift between the upper and lower layers, and each $V_{j}$ represents a scaled combination of zonal flow component $j$ and the squared wave amplitude. Since $a_{j} \sim j^{-4}$ as $j \rightarrow \infty$, Eqs. (1-3) may be well approximated numerically by truncating the sum in Eq. (2) at a finite value $J$, as is done here.

\section{Attractor structure and Lyapunov vectors}

\subsection{The $\gamma=0.1315$ attractor}

A numerical exploration of Eqs. (1-3) for a range of parameter values has been conducted by Pedlosky and Frenzen (1980). Following S2001, the present study focuses on a set of solutions with $m=1$ and $K^{2}=2 \pi^{2}(k=\pi)$, corresponding to a wave with equal zonal and meridional scales, and with friction parameter $\gamma=0.1315$. The results described here were obtained with a truncation at $J=6$ in Eq. (2). The differences between the present numerics and those of Pedlosky and Frenzen (1980) lead to small, but inessential differences in the solutions and their dependence on $\gamma$. The numerical techniques used in the present study are the same as those described in S2001.

For $\gamma=0.1280$ (and for a range of adjacent $\gamma$ ), the numerical solutions approach a limit cycle (S2001, Figs. 1-3). Note that since the Eqs. (1-3) are unchanged by the transformation $(A, B) \rightarrow(-A,-B)$, an asymmetric solution is accompanied by a twin of opposite parity, corresponding to an arbitrary along-channel phase shift of a half-wavelength. For simplicity, attention is restricted here to the solution with parity such that the maximum value of $|A|$ occurs for $A>0$. The corresponding results for the twin solutions may be obtained by changing the appropriate signs or phases.

In the wave-mean oscillation corresponding to this limit cycle, there is a periodic reduction in zonal shear driven by the growing wave, followed by a saturation of wave-growth as the source of the instability is removed, with the subsequent decay of the wave amplitude and a resurgence of the zonal shear. The wave amplitude $A$ oscillates between a positive and a negative maximum, each time remaining small for substantial times as it changes sign. The mean flow correction reduces the vertical shear everywhere, and is nearly in phase with the squared wave amplitude, which is consistent with a dominant balance between mean flow acceleration and potential vorticity fluxes due to secular changes in the wave amplitude, as might be anticipated for small values of friction $\gamma$. Since $\gamma$ is small, the weakly nonlinear phase shift 

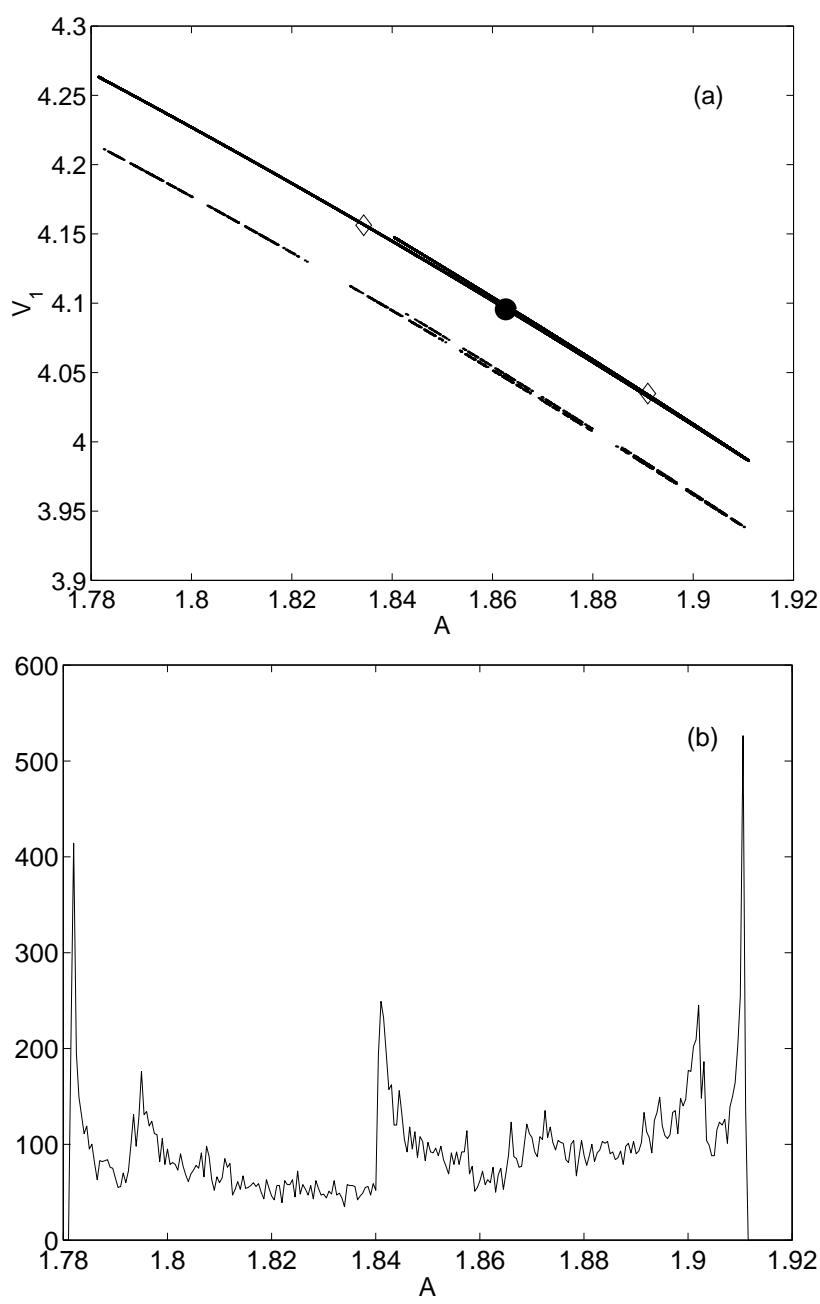

Fig. 1. (a) $A-V_{1}$ phase plane structure of the attractor on the $\{B=0, A>0\}$ Poincaré section, from 25000 points. The large dot shows the location of the lowest-order unstable periodic orbit $p_{1}$. The diamonds show the orientation of the leading Floquet vector of $p_{1}$. The 1993 intersection points of the first 157 unstable cycles are also shown, offset in $V_{1}$ by -0.05 . (b) Histogram of the $A$-values of the 25000 attractor points in (a).

$B \approx d A / d t$ over most of the cycle, as is true for the linear modes of instability of the steady zonal flow.

As $\gamma$ increases past 0.1280 , the system undergoes a sequence of period-doubling bifurcations. Chaos appears to ensue for $\gamma$ greater than about 0.1309 . Here, we focus on the chaotic numerical solution for $\gamma=0.1315$. This is the same value of the friction parameter $\gamma$ for which S2001 studied the properties of the lowest-order unstable periodic orbit, the continuation to $\gamma=0.1315$ of the $\gamma=0.1280$ limit cycle. The solution on the $\gamma=0.1315$ attractor is generally similar to the $\gamma=0.1280$ limit cycle, but with irregular fluctuations in the maximum and minimum amplitude of the oscillation.

The $\gamma=0.1315$ attractor is conveniently analyzed by considering the Poincaré section on the half-plane $\{B=0, A>0\}$. A projection on the $A-V_{1}$ phase plane of a set of points at which a $\gamma=0.1315$ numerical solution intersects this half-plane as shown in Fig. 1a. These points lie nearly along a single curve. The Poincaré map constructed by plotting the $A$-values of successive intersections closely approximates a one-dimensional, single hump map that is asymmetric, but otherwise resembles the logistic map (S2001, Fig. 4).

The following analysis focuses entirely on the structure of the attractor as it is represented in this Poincaré section. An $A-B$ phase plane projection of the continuous $\gamma=0.1315$ attractor time series is shown in Fig. 2b of S2001. The return time between successive Poincaré section points is approximately equal to the period of the lowest-order unstable periodic orbit (24.479), or physically to two weakly nonlinear baroclinic wave life cycles, since each oscillation involves a growth and decay of waves with alternating signs or phases. A histogram of the $A$-values of the points in Fig. 1a is shown in Fig. 1b, and indicates that, with notable exceptions near the maximum and minimum values of $A$ and several intermediate points, the 25000 points of the numerical solution are approximately uniformly distributed in $A$.

\subsection{Lyapunov exponent and vectors}

The leading Lyapunov exponent $\lambda$ and Lyapunov vector $v$ on the attractor were approximated numerically in the standard way (Shimada and Nagashima, 1979; Bennetin et al., 1980): the long-time evolution of an arbitrary, small disturbance to the attractor solution was computed using the linearized equations. The amplification of the linear disturbance on each return to the Poincaré section was computed (using the standard inner-product norm in the $\left\{A, B, V_{j}\right\}$ phase space), and the disturbance was then renormalized each time to prevent unbounded growth. To limit the influence of transients associated with the arbitrary initialization, only the last 25000 of 50000 Poincaré returns are used in the analysis (corresponding to a time series of length $\approx 25000 \times 24.479 \approx 6 \times 10^{5}$, or 50000 weakly nonlinear wave life cycles). Note that this time series is much longer than the length of time over which the numerical solution can track the actual unstable solution from a given initial point with neglible numerical error; it is assumed in the usual way (e.g. a shadowing property) that the numerical results are still meaningful, at least as local and statistical descriptions.

The Lyapunov exponent $\lambda$ was computed as the mean exponential growth rate of the linearized disturbance. During the last 15000 returns, $\lambda=0.01608 \pm 0.00001$, where the error is the standard deviation. For the unstable time-dependent numerical solution on the attractor, this mean exponential growth rate is analogous to the exponential growth rates of normal-mode instabilities of steady flows, as it is the rate at which the fastest growing disturbance will amplify asymptotically in the long-time limit.

The Lyapunov vector $\boldsymbol{v}$ is approximated asymptotically by the successively renormalized linear disturbance at each point on the attractor visited by the numerical solution. For the solution on the attractor, this vector is analogous to the 

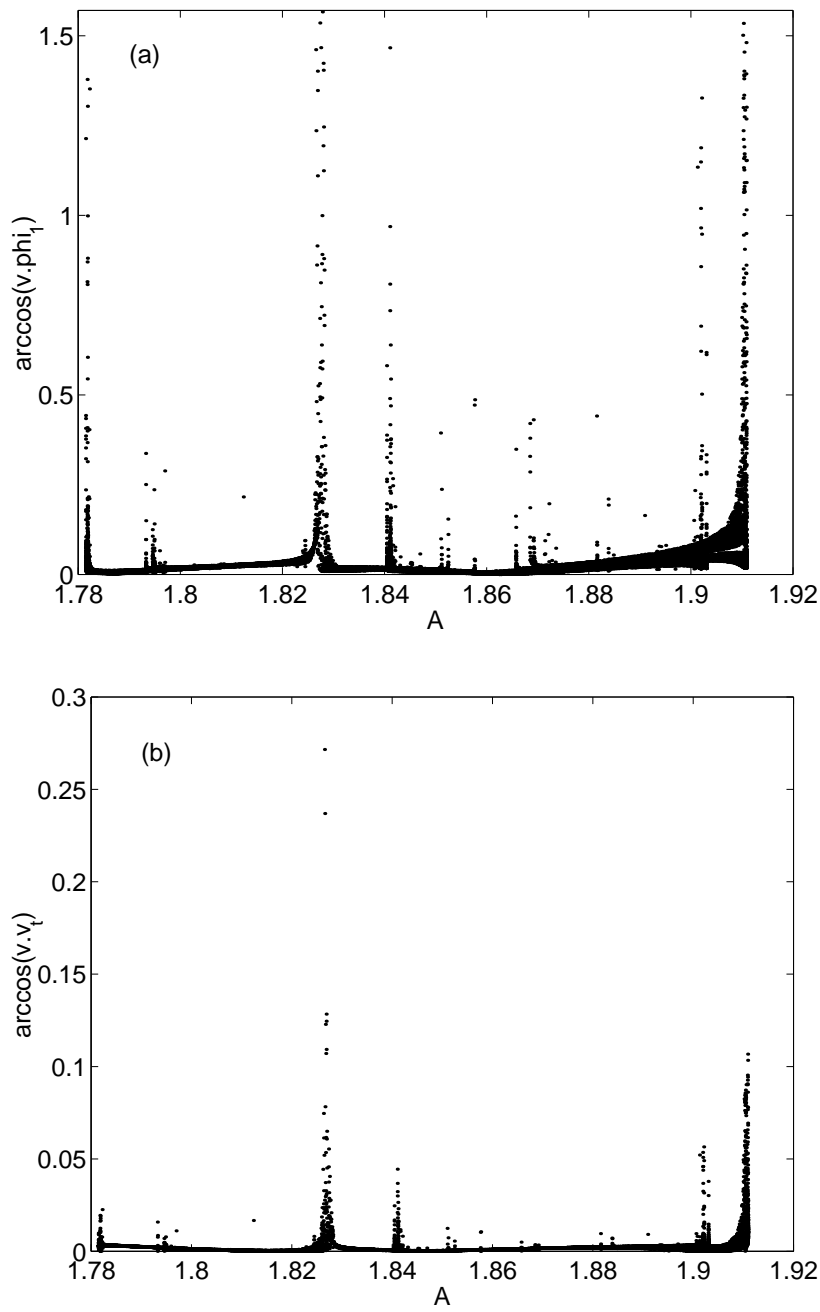

Fig. 2. (a) Relative angles of Lyapunov vectors and the leading Floquet vector $\phi_{1}$ of the lowest-order unstable periodic orbit $p_{1}$, vs. $A$. (b) Relative angles of Lyapunov vectors and the approximate tangent plane to the attractor vs. A. Note the change in vertical scale from (a) to (b).

normal modes of linear instability of steady flows. At each point, any disturbance to the time-dependent numerical solution that is not orthogonal to $v$ will ultimately grow indefinitely under the linearized dynamics, approaching the structure of $\boldsymbol{v}$ asymptotically. Over most of the attractor, $v$ is nearly tangent to the large-scale structure of the attractor. This is indicated in Fig. 2a, in which $\left|\arccos \left(\boldsymbol{v} \cdot \phi_{1}\right)\right|$ (with the standard inner-product, and unit vectors $v$ and $\phi_{1}$ ) is plotted versus $A$ for each Poincaré intersection point, where $\phi_{1}$ is the leading Floquet vector of the lowest-order unstable period orbit $p_{1}$ at $\gamma=0.1315$. The Floquet vector $\phi_{1}$ is, in turn, approximately tangent to the large-scale structure of the attractor, as indicated in Fig. 1a (see also S2001). Figure 2a also indicates that $\phi_{1}$ provides a good approximation of $v$ over most of the attractor. The physical structure of $\phi_{1}$ is shown in Fig. 9b of S2001.

A planar approximation to the large-scale attractor structure at the Poincaré section can be constructed from the tan-
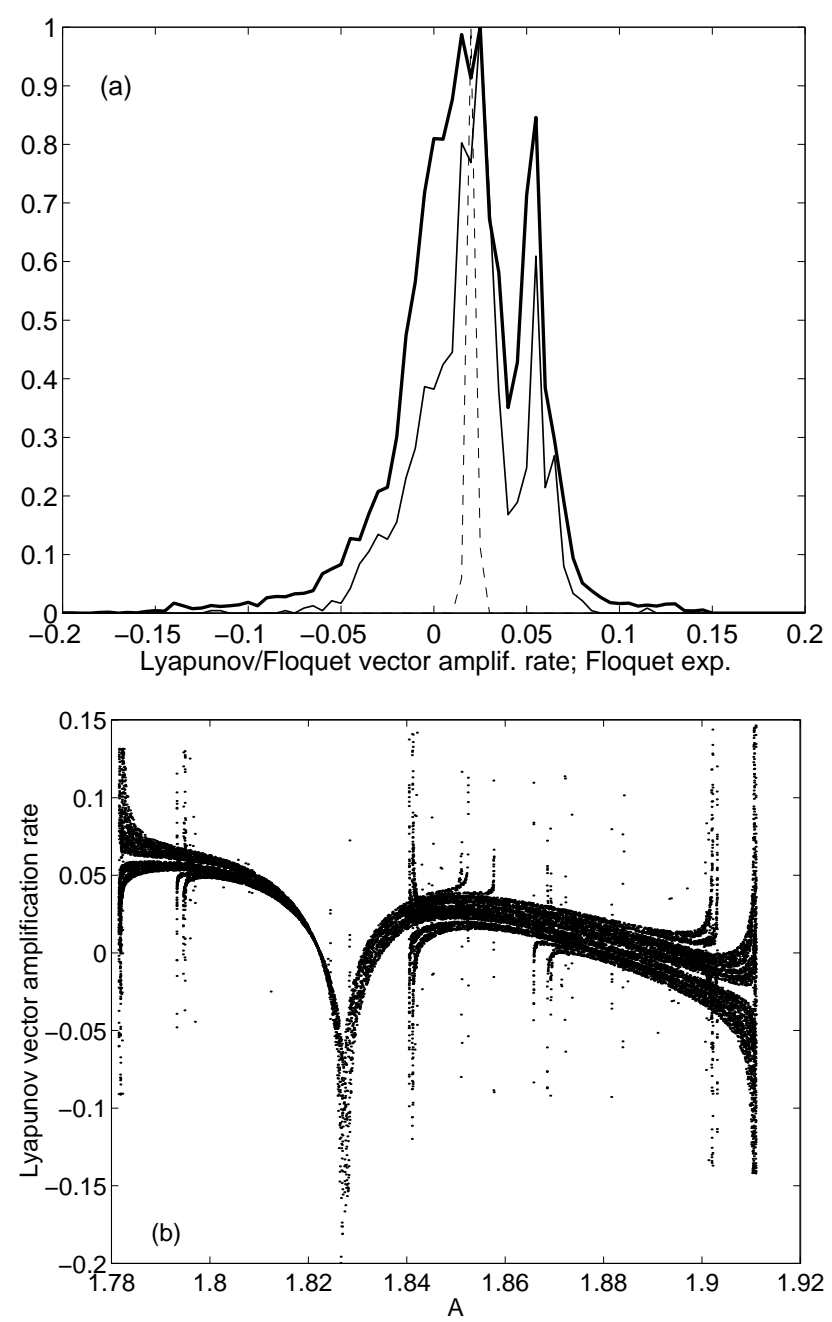

Fig. 3. (a) Normalized histograms of Poincaré return exponential growth rates $\lambda_{T}$ and $\lambda_{1 T}$ of Lyapunov (thick solid line) and leading Floquet (thin) vectors, respectively, and leading Floquet exponents $\lambda_{1}$ (dashed). (b) Poincaré return exponential growth rates $\lambda_{T}$ of Lyapunov vectors vs. $A$.

gent $\phi_{2}$ to $p_{1}$ and a linear approximation to the attractor on the section, in which the coordinates $\left\{V_{j}\right\}$ are parameterized in terms of $A$ (for example, $V_{1}=-2.1379 A+8.0763$; compare to Fig. 1a). The Lyapunov vector $\boldsymbol{v}$ is essentially tangent to this plane over almost all of the section (Fig. 2b). Most of the large angles between $\boldsymbol{v}$ and $\phi_{1}$ arise when the Lyapunov vector rotates away from $\phi_{1}$ but remains within the large-scale tangent plane to the attractor. Thus, the Lyapunov vector does provide, for this relatively simple attractor geometry, a useful guide to the distribution of nearby states on the attractor.

Despite this near uniformity across the attractor of the Lyapunov vector orientation, the individual Poincaré return growth rates $\lambda_{T}$, from which the mean growth rate $\lambda$ was computed, fluctuate over a range that is an order of magnitude larger than $\lambda$, from growth rates as large as 0.14 , to decay rates as large as -0.2 (Fig. 3). This result is consistent with the well-known slow convergence of Lyapunov expo- 
nents computed in this manner. The $\lambda_{T}$ are the mean exponential rates of growth of the Lyapunov vector $\boldsymbol{v}$ (in the standard norm) during each return to the Poincaré section, computed according to $\lambda_{T}=\ln (|\boldsymbol{v}(t+T)| /|\boldsymbol{v}(t)|) / T$, prior to renormalization of $\boldsymbol{v}(t+T)$, where $T \approx 24$ is the return time. The $\lambda_{T}$ are bimodally distributed, with a broad peak near $\lambda$ and a distinct secondary peak near 0.05 (Fig. 3a). Many of the $\lambda_{T}$ are as small as -0.02 or as larger as 0.05 ; these variations arise over large parts of the attractor despite the small departures of $v$ from $\phi_{1}$ (Fig. 3b); for comparison, $\phi_{1}$ has Floquet exponent (see below) $\lambda_{1} \approx 0.025$, slightly larger than $\lambda$.

Extreme values of $\lambda_{T}$ occur where $\boldsymbol{v}$ is the farthest from the tangent to $\phi_{1}$, or the attractor (Figs. 2, 3a, 4a, 4c). All (relatively) large departures of $\boldsymbol{v}$ from these tangencies are associated with a decay of $v\left(\lambda_{T}<0\right)$; as tangency with $\phi_{1}$ is approached, $\lambda_{T}$ approaches $\lambda_{1} \approx 0.025$ (Fig. 4b). The smallest $\lambda_{T}$ are found near $A=1.826$ (Fig. 3b), consistent with the peak and vanishing slope of the approximate onedimensional map at this point. Most of the positive values of $\lambda_{T}$ are found in $A<1.81$, while most $\lambda_{T}$ are negative for $A>1.81$. Thus, the disturbances described by the Lyapunov vector tend to amplify only when the wave amplitude $A$ is small, and they decay when $A$ is large or near the critical value of 1.826 . This is consistent with the general character of the oscillation, in which nonlinear (large $A$ ) stabilizing mechanisms arrest the growth of the linear (small $A$ ) instability.

\section{Periodic orbits and Floquet vectors}

One approach to the study of the structure and dynamics of chaotic systems involves the analysis of an associated set of unstable periodic orbits (e.g. Cvitanović et al., 2000). This analysis is simplified if a symbolic dynamics can be identified that relates the orbits to symbol sequences. As noted above, in the present case, the evolution for $\gamma=0.1315$ may be usefully represented by a one-dimensional map (S2001, Fig. 4). From the spline representation of this map, unstable period orbits were determined in the standard way by associating the symbols 0 and 1 with the intervals to the left and right, respectively, of the point where the map achieves its maximum $A=1.826$, generating a set of binary symbol sequences, and finding the corresponding unique orbit points by inverse iteration. These points were then used as first guesses for the periodic points of the differential equations, which were improved using Newton's method. The result of this set of calculations is a set of unstable periodic orbits that are related in an essential way to the attractor dynamics and "fill out" (more precisely, and under certain conditions that may or may not strictly hold here, are dense on) the attractor. For some examples of these orbits, see Figs. 5 and 9a of S2001.

A list of all symbol sequences up to length 15 for which unstable cycles were computed for $\gamma=0.1315$ is given in Table 1 . There are a total of 157 such cycles. In the present
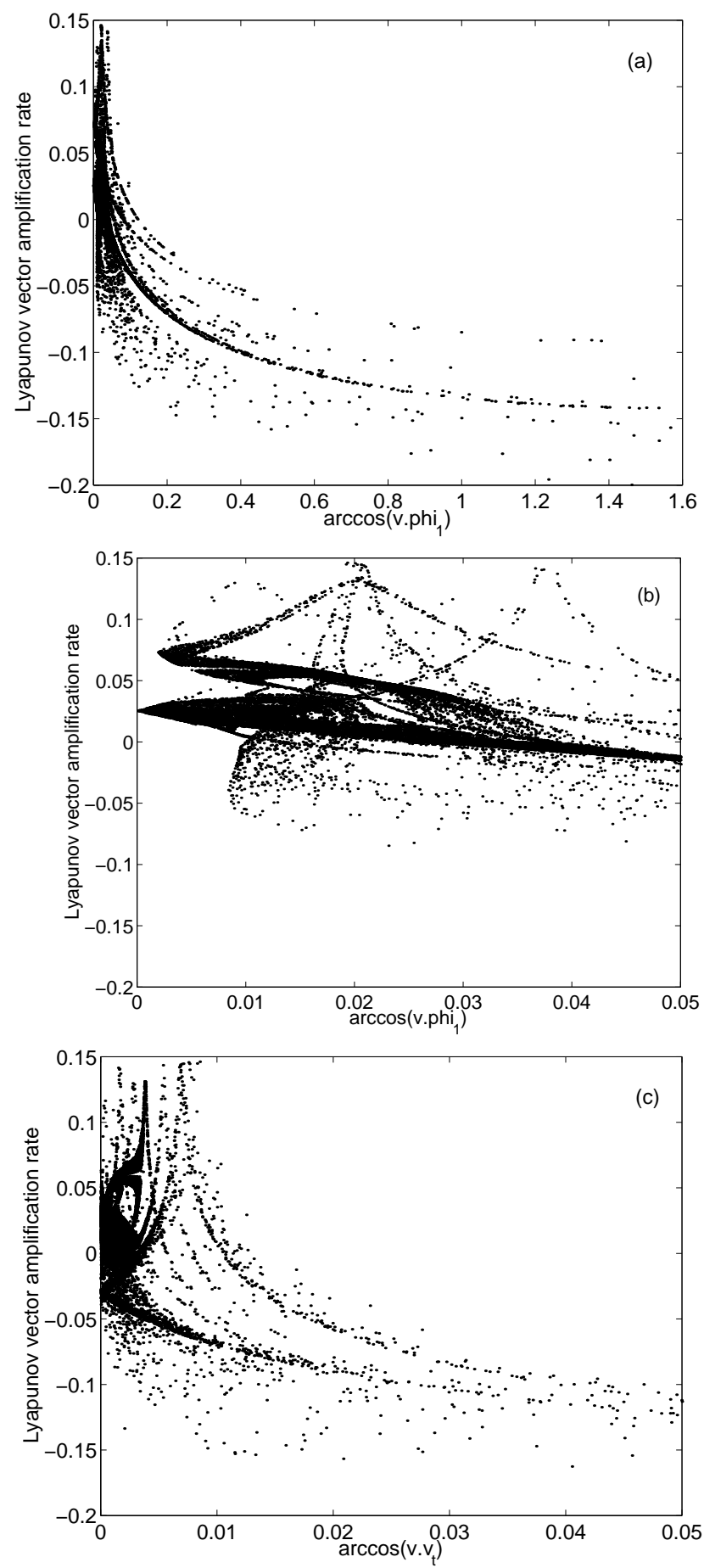

Fig. 4. (a) Scatter plot of Poincaré return exponential growth rates $\lambda_{T}$ vs. relative angles of Lyapunov vectors and the leading Floquet vector $\phi_{1}$ of the lowest-order unstable periodic orbit $p_{1}$. (b) Same as (a), but with expanded scale for small angles. (c) Scatter plot of Poincaré return exponential growth rates $\lambda_{T}$ vs. relative angles of Lyapunov vectors and the approximate tangent plane to the attractor.

case, due to the geometry of the map, many possible symbol sequences do not have corresponding cycles. For example, no cycle corresponds to the repeated sequence 0 , nor to any 

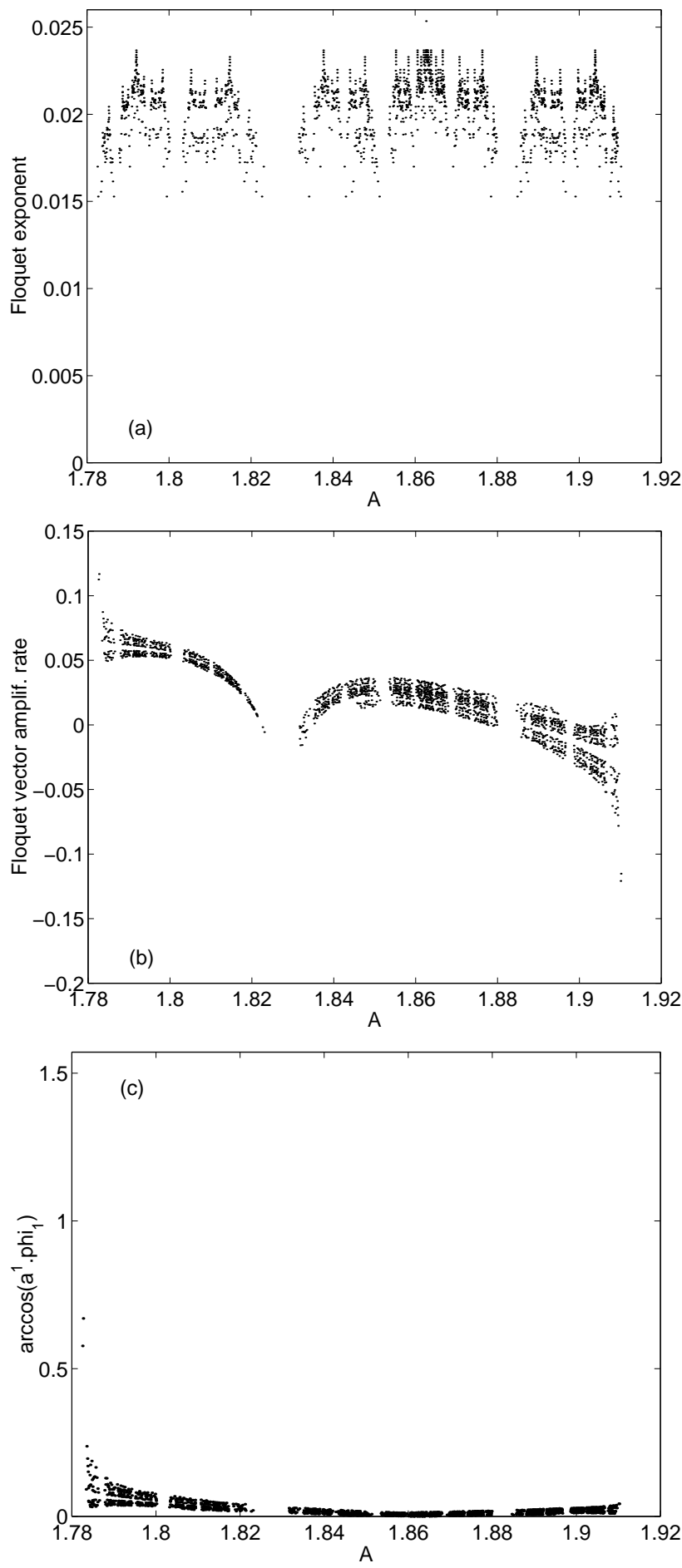

Fig. 5. (a) Floquet exponents for 157 unstable cycles, plotted vs. $A$ at each of the 1993 intersection points of the 157 cycles with the Poincaré section. (b) Poincaré return exponential growth rates $\lambda_{1 T}$ of the leading Floquet vector vs. A, for each of the 157 cycles. (c) Relative angles of the leading Floquet vector for each cycle and the leading Floquet vector $\phi_{1}$ of the lowest-order unstable periodic orbit $p_{1}$.

sequence containing 00 , since all points in the left-hand interval are mapped to points in the right-hand interval. The 1993 points where these 157 cycles intersect the Poincaré section are shown in Fig. 1a, with $V_{1}$ values offset by -0.05 . The coverage of the attractor is not uniform, and there are several large gaps, for example, near $A=1.82$ and $A=1.88$.

The solutions of the linearized equations for small disturbances to these unstable cycles may be computed by standard techniques for linear differential systems with periodic coefficients (e.g. Coddington and Levinson, 1955), often known as Floquet theory, as described in detail in S2001. The solutions are obtained for the truncation $J=6$, so the result of each of these calculations is a set of 8 time-dependent normal-modes or "Floquet vectors" $\left\{\phi_{j}, j=1, \ldots, 8\right\}$ and 8 corresponding Floquet exponents $\left\{\lambda_{j}, j=1, \ldots, 8\right\}$; the $\lambda_{j}$ are the mean exponential growth (or decay) rates of the corresponding disturbance over the cycle length. Note that the exponents $\lambda_{j}$ will, in general, differ from cycle to cycle, but for simplicity, the dependence on cycle is dropped from the notation here. Except for the exponential growth factors, each of these Floquet vectors is also periodic, with the period equal to the cycle period (or twice the cycle period). For an example of the physical structure of one of these Floquet vectors, see Fig. 9b of S2001.

The Floquet exponents for these 157 cycles are distributed essentially in same way as those shown in Fig. 8 of S2001: for each cycle, there is one growing mode $\left(\lambda_{1}>\right.$ 0 ), one neutral mode $\left(\lambda_{2} \approx 0\right)$, and six decaying modes $\left(\lambda_{j}<0, j=3, \ldots, 8\right)$. The neutral $(j=2)$ and damped zonal flow $(j=4, . ., 7)$ modes have $\lambda_{j}$ nearly independent of cycle, while the values of $\lambda_{1}, \lambda_{3}$, and $\lambda_{8}$ fluctuate. The distribution of $\lambda_{1}$ for the 157 cycles is shown in Fig. 3a; the maximum and minimum values of $\lambda_{1}$ are 0.0253 (for the $p_{1}$ cycle) and 0.0153 (for a 12-cycle with sequence 110111011010).

If the leading Floquet exponent $\lambda_{1}$ for each cycle is plotted at the Poincaré section points of the corresponding cycle, the resulting distribution is not smooth (Fig. 5a). The smallest $\lambda_{1}$ tends to occur at the edges of the gaps in coverage noted above, suggesting the presence of weakly unstable cycles of longer cycle length. This hypothesis is consistent with the relatively small value of the Lyapunov exponent $\lambda$ computed above, compared to most of the first 157 leading Floquet exponents $\lambda_{1}$; according to the cycle expansion theory, $\lambda$ should be accurately approximated by a suitable average of the $\lambda_{1}$, weighted inversely by stability.

The leading Floquet vectors of higher-order cycles are, in general, nearly parallel to the leading Floquet vector $\phi_{1}$ of the lowest-order cycle $p_{1}$ (Fig. 5c). Individual Poincaré return growth rates $\lambda_{j T}$ of the Floquet vectors were computed in the same manner as the Lyapunov vector growth rates $\lambda_{T}$; they are the mean exponential growth or decay rates of the corresponding Floquet vector during each return to the Poincaré section. The amplification factors $\lambda_{1 T}$ of the leading Floquet vectors show a pattern (Fig. $5 \mathrm{~b}$ ) that is strikingly similar to that of the Lyapunov vector growth rates $\lambda_{T}$ (Fig. 3b). The distribution of the $\lambda_{1 T}$ from the first 157 cycles (Fig. 3a) closely resembles the distribution of the $\lambda_{T}$; note that it reproduces the secondary peak near 0.05 . Evidently, only the leading Floquet vectors of the higher-order cycles are re- 
Table 1. Symbol sequences for first 157 unstable cycles for $\gamma=0.1315$

\begin{tabular}{|r|r|r|r|r|}
1 & 11110111010 & 1111010111010 & 11011101111010 & 11111111101010 \\
10 & 11110101110 & 111110111010 & 1111010111110 & 110101111011010 \\
1110 & 11111111010 & 1111010111110 & 11111010111110 & 110101110111010 \\
11010 & 11111101110 & 1111011111010 & 11110111111010 & 110101111111010 \\
11110 & 11110111110 & 1111110101110 & 11111110101110 & 110101110101110 \\
111010 & 11111111110 & 111111111010 & 1111111111010 & 110101111101110 \\
1101010 & 111010101010 & 1111011101110 & 11111011101110 & 110101111011110 \\
1111010 & 111110101010 & 1111111101110 & 11110111101110 & 110101110111110 \\
1111110 & 111010101110 & 1111110111110 & 11110111111110 & 11111010111010 \\
11101010 & 111111101010 & 1111111111110 & 11111110111110 & 111101110111010 \\
11111010 & 110101111010 & 11101010101010 & 11111111111110 & 111101110101110 \\
11111110 & 110111011010 & 11111010101010 & 110101010101010 & 111111110111010 \\
110101010 & 110101111110 & 11010101011010 & 111101010101010 & 111101011111010 \\
111101010 & 111110111010 & 11010101011110 & 110101010101110 & 111101011101110 \\
110101110 & 111101111010 & 11101010101110 & 111111010101010 & 111101011111110 \\
111111010 & 111110101110 & 11111110101010 & 110101010111010 & 11111011111010 \\
111101110 & 111111111010 & 11010101111010 & 110101010111110 & 1111101011110 \\
111111110 & 111111101110 & 11101011101010 & 110101110101010 & 111101111111010 \\
1110101010 & 111101111110 & 11111011101010 & 111101110101010 & 111111110101110 \\
1111101010 & 111111111110 & 11010111101010 & 111101010101110 & 111111111111010 \\
1101011110 & 1101010101010 & 11110111101010 & 11111110101010 & 11111011101110 \\
1110101110 & 1111010101010 & 11111010101110 & 110101011101010 & 111101110111110 \\
1111111010 & 1101010101110 & 11111111101010 & 110101011111010 & 111101111101110 \\
1111101110 & 1111110101010 & 11010111011010 & 110101011101110 & 111111111101110 \\
1111111110 & 1101010111010 & 11010111111010 & 111101011101010 & 11110111111110 \\
11010101010 & 1101011101010 & 11010111101110 & 11111011101010 & 1111111011110 \\
11110101010 & 1111011101010 & 11010111011110 & 111101010111010 & 111111011111110 \\
11010101110 & 1111010101110 & 11010111111110 & 111101010111110 & 111111111111110 \\
11111101010 & 1111111101010 & 11111010111010 & 110101111101010 & \\
11010111010 & 1101011101110 & 11101011101110 & 111101111101010 & \\
11010111110 & 1101011111110 & 11111110111010 & 111111010101110 & \\
& & 1110111110 & 111111101110 & 1101011111110 \\
& & & \\
11010 &
\end{tabular}

quired to describe most of the transient growth and decay of the Lyapunov vector.

The distribution of the Floquet vector amplifications $\alpha_{j}=$ $e^{\lambda_{j T} T}$ generally follows the pattern of the distributions of Floquet exponents $\lambda_{j}$, though with a greatly increased spread (Fig. 6). Modes 2 and 4-7 have narrow amplification distributions, as do their exponents, while modes 1,3 and 8 have broad distributions. This is consistent with the observation in S2001 that the dynamical splitting between wave-dynamical and damped zonal flow modes observed in the lowest-order cycles persists in the higher-order cycles, and further suggests that it persists even during transient growth and decay segments of the higher-order cycles.

\section{Singular vectors}

The Lyapunov vector, described above, describes the characteristic structure of disturbances that amplify indefinitely under the linear dynamics, in a way that is analogous to normalmode instability for steady flows. However, it generally does not describe the linear disturbances that are maximally amplified after a fixed interval of time. Such optimal distur- bances, referred to here as singular vectors (SVs), generally exhibit transient growth, and do not have fixed modal structure (e.g. Lorenz, 1965; Farrell, 1989). In this section, SVs are computed on the 157 unstable cycles discussed in the previous section. As shown above, the leading Floquet vectors from these 157 cycles are sufficient to represent much of the detailed time-evolution of the Lyapunov vector over most of the attractor. It is presumed here that SVs based on the complete set of Floquet vectors from these 157 cycles are sufficient to describe the general characteristics of SVs on the attractor in a similar way.

Consistent with the preceding analysis, SVs are computed here for a single optimization interval (slightly different in duration for each cycle), corresponding to the first Poincaré return from each Poincaré section point on each cycle. This is the same set of intervals that was used above to calculate the Lyapunov and Floquet vector transient growth rates $\lambda_{T}$ and $\lambda_{j T}$. The standard inner-product norm in the $\left\{A, B, V_{j}\right\}$ phase space is used to compute the SVs; this is similar to, but slightly different from the "AE" norm used in S2001. Results for the SVs will depend on the norm used, but in S2001, the qualitative results were relatively insensitive to this choice. 


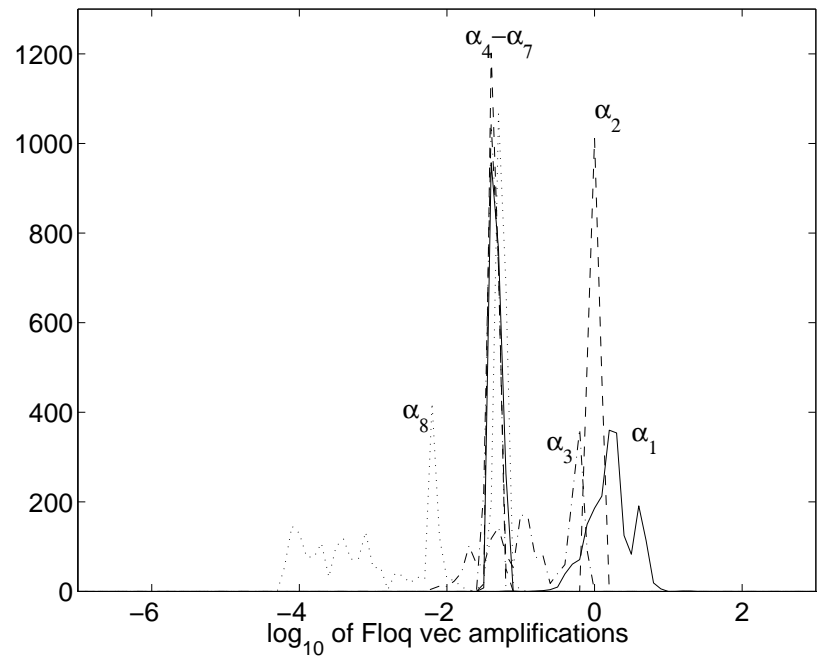

Fig. 6. Histogram of the base-10 logarithm of the Poincaré return amplification $\alpha_{j}=e^{\lambda_{j T} T}$ of Floquet vector $j, j=1, \ldots, 8$ for each intersection point of each cycle.

The SVs are otherwise computed in each case in exactly the same manner as in S2001.

The distributions of the singular values $\mu_{j}, j=1, \ldots, 8$ for each of the 1993 sets of 8 SVs is shown in Fig. 7. Only the first SV is strongly amplifying. The second is approximately neutral in most cases, the third is mostly decaying, and the fourth through eighth are always decaying. In general, these singular values are consistent with those computed for the lowest-order orbit $p_{1}$ in S2001. The first SV amplifies more rapidly than the first Floquet vector, and the eighth SV decays more rapidly than the eighth Floquet vector, while the intermediate SV decay rates follow the corresponding Floquet decay rates closely.

The decompositions of the 1993 leading SVs in terms of the Floquet vectors for the corresponding cycle also show a qualitatively similar pattern to that found in S2001 for the lowest-order orbit (Fig. 8). The leading SVs are dominated by the wave-dynamical modes $(j=1,2,8)$, with some contribution from the intermediate $(j=3)$ mode. The projections of the leading SVs on the decaying zonal mean modes $(j=4, \ldots, 7)$ are uniformly small.

\section{Discussion}

The present analysis of disturbance growth for weakly nonlinear baroclinic waves extends a recent study (S2001), which considered only simple, stable and unstable timeperiodic basic states, to the case of a chaotic basic state. Despite the qualitative difference in the time-dependence of the underlying basic states, the present results are largely consistent with the results of S2001.

The Lyapunov vector and exponent are the natural conceptual extensions to the chaotic basic state of the normal modes

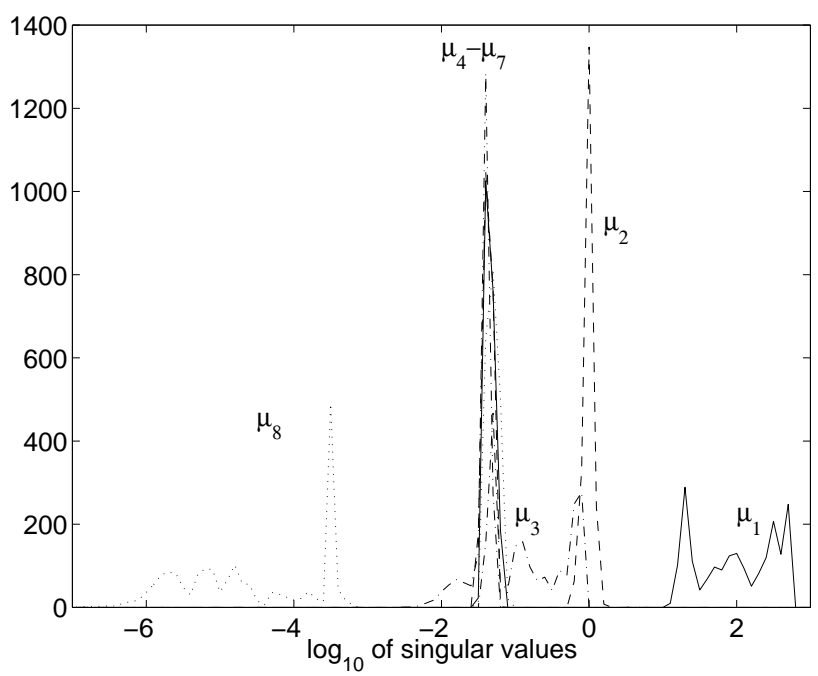

Fig. 7. Histogram of the base-10 logarithm of the Poincaré return singular value $\mu_{j}$ for singular vector $j, j=1, \ldots, 8$ for each intersection point of each cycle.

and growth rates of linear instabilities of steady basic states, and of the time-dependent Floquet normal modes and growth rates of linear instabilities of time-periodic basic states. For the chaotic basic state, the Lyapunov vector is also the simplest analog to the bred modes of numerical bred modes of numerical weather prediction. Over most of the attractor, the Lyapunov vector closely resembles the unstable Floquet normal mode $\phi_{1}$ of $p_{1}$, the lowest-order unstable periodic orbit analyzed in S2001. In turn, $\phi_{1}$ closely resembles the neutral mode $\phi_{2}$ that is tangent to $p_{1}$, the basic-state oscillation (S2001). Thus, as in S2001, disturbance growth is dominated by the same baroclinic wave processes that control the evolution of the basic state. The Lyapunov exponent $\lambda \approx 0.016$ is of the same order but smaller than the leading Floquet exponent $\lambda_{1} \approx 0.025$ of $p_{1}$.

Transient growth and decay of the Lyapunov vector occurs on the baroclinic wave time scale with exponential rates that are an order of magnitude larger than $\lambda$. These events involve only minor changes in the structure of the Lyapunov vector, which remains nearly parallel to $\phi_{1}$ over most of the attractor. They are accurately represented by the corresponding transient growth and decay of the leading Floquet vectors of the higher-order unstable cycles. Thus, even for these transient events, only the growing modes of the unstable cycles are essentially sufficient to describe the structure and evolution of the Lyapunov vector.

The Floquet vectors of the higher-order unstable cycles split into wave-dynamical and decaying zonal flow modes. Singular vectors, computed using the standard norm on the baroclinic wave time scale for the set of 157 unstable cycles, reflect this splitting: the leading singular vectors are dominated by the wave-dynamical Floquet modes. Similar results were reported in S2001 for $p_{1}$. The maximum singular value amplifications are two orders of magnitude larger than the 


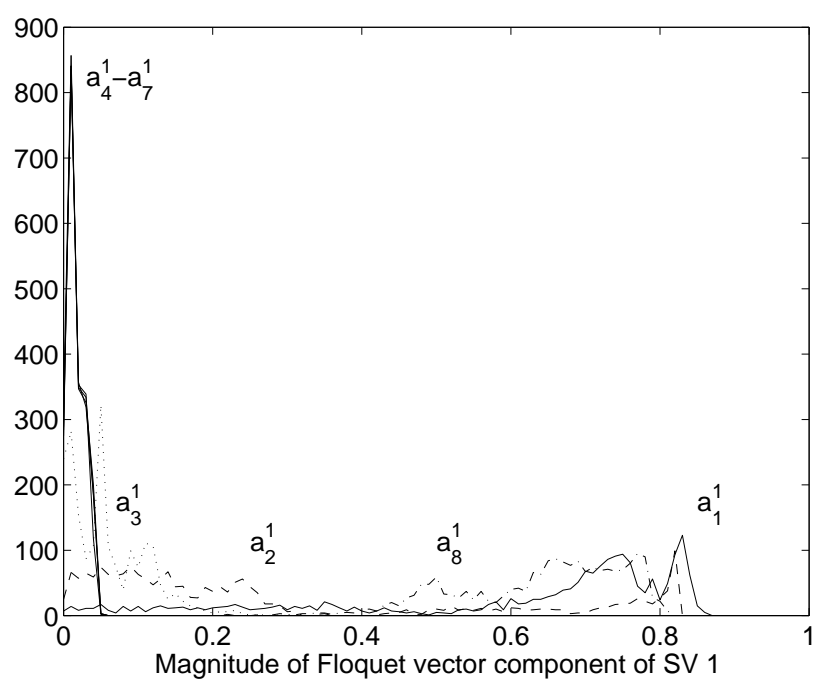

Fig. 8. Histogram of the magnitudes of the Floquet vector components $a_{j}^{1}, j=1, \ldots, 8$ of the leading Poincaré return singular vector $\mathbf{a}^{1}$ for each intersection point of each cycle.

corresponding maximum transient amplifications of the Floquet modes of the unstable cycles, but there is typically only one amplifying singular vector, corresponding to the single amplifying Floquet mode. This is true despite that the optimization interval was much shorter than the periods of many of the higher-order cycles.

These results demonstrate that singular vectors and the extensions of normal-mode instabilities for this irregular, timedependent flow are closely related, as they were found to be in S2001 for the time-periodic basic states. The Lyapunov vector, the simplest analog of bred modes, captures the same disturbance structures and transient growth and decay events that dominate the singular vectors, with one important exception: the decaying, inviscidly-damped, wavedynamical Floquet vector is a central element of the growing singular vectors, but is essentially absent from the Lyapunov vector. The structure of the decaying wave-dynamical mode generally resembles that of the other wave-dynamical modes, which results in a tendency toward non-orthogonality that the singular vector exploits to produce a large transient amplification. Evidently, the damped zonal flow modes are sufficiently different in structure from the wave-dynamical modes that they have a negligible contribution to the growing singular vectors.

It is perhaps surprising that the leading Lyapunov and Floquet vectors, here and in S2001, have a structure similar to the tangent to the evolving basic-state flow. As noted above, this indicates that the processes that control disturbance growth in this model are essentially the same as those that control the evolution of the basic state. In a more complex model that admits a wider range of scales and physical processes, or in a similar model but farther from marginal stability of the basic cycle, the similarity between the unsta- ble modes and the tangent to the flow may not persist. Thus, and in general, the degree to which the present results will extend to more complex models is uncertain.

The present results are generally consistent with the analysis by Trevisan and Pancotti (1998) of Lyapunov, Floquet, and singular vectors for a low-order unstable periodic cycle of the three-component Lorenz (1963) model. Those authors also find that the unstable Floquet mode tends to resemble the tangent along the cycle. Presumably, this would also be true for the higher order cycles of the Lorenz model, as it is here. They also find, as here, that rapidly amplifying singular vectors arise as a consequence of non-orthogonality of the Floquet modes.

\section{Summary}

This study has addressed the mechanisms of growth of linear disturbances to an irregular, chaotic basic state in a simple model of weakly nonlinear baroclinic wave-mean interaction. It extends the previous results of S2001, which considered disturbances to time-periodic basic states of the same model. Many of the present conclusions are similar to those of S2001. Disturbance growth in this simple model was found to be related to the wave growth and decay mechanisms associated with the time-dependent basic state. Floquet vectors of the higher-order unstable cycles were found to divide into two dynamical classes, the first associated with baroclinic wave dynamics and the second with the frictional decay of high meridional modes of the zonal flow. The decompositions of the leading singular vector in terms of the time-dependent Floquet vectors of the higher-order cycles generally reflected this dynamical split, with the wavedynamical components dominating.

In addition, the Lyapunov vector, which for the chaotic basic state in this model is the simplest analog of the bred modes used in numerical weather prediction, was shown to capture most of the transient variability of the growing wavedynamical Floquet vectors. Since these, along with the tangent to the orbit itself, are two of the three dominant components of the singular vectors, the present results suggest a close relation between bred modes and singular vectors. The primary difference between the two sets of modes is the contribution of the decaying wave-dynamical mode to the singular vectors. This confirms one of the hypotheses of S2001 for the nonperiodic state in this simple model. If this result extends to more complex models, and the presence of decaying wave-dynamical modes are found generally to distinguish singular vectors from bred modes, then a practical question that arises in comparing ensemble generation methods is, are the additional decaying wave-dynamical modes valuable to ensemble generation in operational forecasting implementations?

Acknowledgement. This research was supported by the Office of Naval Research, Grant N00014-98-1-0813. I am grateful to E. Spiegel for helpful conversations on periodic orbit theory at the 
1997 Summer School on Geophysical Fluid Dynamics at the Woods Hole Oceanographic Institution.

\section{References}

Artuso, R., Aurell, E., and Cvitanović, P.: Recycling of strange sets I: Cycle expansions, Nonlinearity, 3, 325-359, 1990a.

Artuso, R., Aurell, E., and Cvitanović, P.: Recycling of strange sets II: applications, Nonlinearity, 3, 361-386, 1990b.

Bennetin, G., Galgani, L., Giorgilli, A., and Strelcyn, J.-M.: Lyapunov characteristic exponents for smooth dynamical systems and for Hamiltonian systems: A method for computing all of them, Meccanica, 15, 9-21 1980.

Buizza, R.: The impact of orographic forcing on barotropic unstable singular vectors, J. Atmos. Sci., 52, 1457-1472, 1995.

Buizza, R. and Palmer, T.: The singular vector structure of the atmospheric general circulation, J. Atmos. Sci., 52, 1434-1456, 1995.

Buizza, R., Tribbia, J., Molteni, F., and Palmer, T.: Computation of unstable structures for a numerical weather prediction model, Tellus, 45A, 388-407, 1993.

Christiansen, F., Cvitanović, P., and Putkaradze, V.: Spatiotemporal chaos in terms of unstable recurrent patterns, Nonlinearity, 10, 55-70, 1997.

Coddington, E. and Levinson, N.: Theory of Ordinary Differential Equations, McGraw-Hill, New York, 1955.

Cvitanović, P., Artuso, R., Mainieri, R., and Vatay, G.: Classical and Quantum Chaos, http://www.nbi.dk/ChaosBook/, Niels Bohr Institute, Copenhagen, 2000.

Ehrendorfer, M. and Tribbia, J.: Optimal prediction of forecast error covariances through singular vectors, J. Atmos. Sci., 54, 286313, 1997.

Epstein, E.: Stochastic dynamic prediction, Tellus, 21, 739-759, 1969.

Farrell, B.: Optimal excitation of baroclinic waves. J. Atmos. Sci., 46, 1193-1206, 1989.
Klein, P. and Pedlosky, J.: A numerical study of baroclinic instability at large supercriticality, J. Atmos. Sci., 43, 1243-1262, 1986.

Legras, B. and Vautard, R.: A guide to Lyapunov vectors. Proceedings 1995 ECMWF Seminar on Predictability, Vol. I, 143-156, 1996.

Leith, C.: Theoretical skill of Monte Carlo forecasts, Mon. Wea. Rev., 102, 409-418, 1974.

Lorenz, E.: Deterministic nonperiodic flow, J. Atmos. Sci., 12, 130$141,1963$.

Lorenz, E.: A study of the predictability of a 28-variable atmospheric model, Tellus, 17, 321-333, 1965.

Pedlosky, J.: Finite-amplitude baroclinic waves with small dissipation, J. Atmos. Sci., 28, 587-597, 1971.

Pedlosky, J.: Geophysical Fluid Dynamics, Springer-Verlag, New York, 1987.

Pedlosky, J. and Frenzen, C.: Chaotic and periodic behaviour of finite-amplitude baroclinic waves, J. Atmos. Sci., 37, 11771196, 1980.

Samelson, R.: Periodic orbits and disturbance growth for baroclinic waves, J. Atmos. Sci., 58, 436-450, 2001.

Shimada, I. and Nagashima, T.: A numerical approach to ergodic problem of dissipative dynamical systems, Prog. Theor. Phys.,61, 1605-1616, 1979.

Szunyogh, I., Kalnay, E., and Toth, Z.: A comparison of Lyapunov and optimal vectors in a low resolution GCM, Tellus, 49A, 200 227, 1997.

Toth, Z. and Kalnay, E.: Ensemble forecasting at NCEP and the breeding method, Mon. Wea. Rev., 125, 3297-3319, 1997.

Trevisan, A. and Legnani, R.: Transient error growth and local predictability: a study in the Lorenz system, Tellus, 47A, 103-117, 1995.

Trevisan, A. and Pancotti, F.: Periodic orbits, Lyapunov vectors, and singular vectors in the Lorenz system, J. Atmos. Sci., 55, 390-398, 1998.

Vannitsem, S. and Nicolis, C.: Lyapunov vectors and error growth patterns in a T21L3 quasigeostrophic model, J. Atmos. Sci., 54, 347-361, 1997. 